\title{
STUDIES OF ACCOMMODATION OF NERVE IN PARATHYROID DEFICIENCY
}

\author{
BY ROBERT S. WIGTON ${ }^{1}$ AND FRANK BRINK, JR. \\ (From the Eldridge Reeves Johnson Research Foundation and the Institute of Neurology, \\ University of Pennsylvania, Philadelphia)
}

(Received for publication April 13, 1944)

The properties of human nerve change with variations in the chemical content of the body fluids. When this change is pronounced, the altered condition of the nervous tissue is readily observed, as in tetany due to hypocalcemia or marked alkalosis. A quantitative measure of the condition of the nerves could be used to determine less obvious but perhaps significant variations in the functional characteristics of nerve. Such measurements would reveal the graded changes in nerve tissue associated with the gradual changes in the blood chemistry. The purpose of the present studies is to test the usefulness of a quantitative index of the changes in human nerve that accompany variations in the calcium ion concentration in the body fluids. The property of nerve employed is the rate of accomodation to an electrical current.

The process of accomodation can be described briefly as follows. The minimum constant current which just stimulates a nerve is called the rheobase. When the stimulating current rises slowly to its final value, the threshold strength of the current is greater than the rheobase. The slower the rate of rise of the current the higher is the threshold value. The property of a nerve by which it thus adjusts to the gradual development of the stimulating action of an electric current is called accomodation.

This property is markedly altered in excised nerves by variations in the concentration of ionized calcium in the fluids bathing the tissue (1). Furthermore, it can be readily measured in man as has been shown (1). An opportunity to study the variations in this property of human nerve in 5 cases of hypoparathyroidism was provided through the cooperation of the Medical Service of the Hospital of the University of Pennsylvania. ${ }^{2}$

1 With the financial assistance of the Kirby-McCarthy Fund.

2 These cases of post-thyroidectomy parathyroid deficiency were being studied by Dr. Edward Rose and
A test was being made of dihydrotachysterol, or A T 10, a drug used in the treatment of hypoparathyroidism. Complete calcium balance studies were made so that the quantitative measures of the functional state of the peripheral nerve could be compared with chemical changes.

Observations were made during a control period when large doses of calcium lactate and viosterol were given. The patients were then taken off all medication for periods of 7 to 12 days during which time they developed symptoms and signs of hypocalcemia. These manifestations consisted of numbness and tingling of the hands and feet, intermittent carpo-pedal spasm, and positive Chvostek and Trousseau signs. Upon administration of $\mathrm{A} T 10$, their blood calcium level rose and symptoms of tetany disappeared. The functional state of the peripheral nerve was tested at intervals by determinations of the property of accomodation.

\section{APPARATUS}

The apparatus for the determination of accommodation of nerve was designed to deliver a current of rectangular wave form (i.e., instantly rising) to be used for the determination of the rheobase, and exponentially increasing currents of various specified rates of rise for the determination of the threshold of the nerve to a series of slowly rising currents.

Approximate determination of the threshold of a nerve to currents passed through electrodes applied to the skin is readily achieved with practical values of current, if the cathode is placed near the nerve at a point where it lies close to the surface. An accurate determination of the threshold, however, would require a knowledge of the fraction of the current actually passing through the nerve fibers. This is practically impossible to determine, and for the present purpose it is unnecessary to do so. By a determination of the ratio between the threshold value of a slowly rising current and the rheobase a measure is obtained which is independent of the actual

Dr. F. W. Sunderman. A report of their clinical and chemical studies has been published. (Effect of dihydrotachysterol in treatment of parathyroid deficiency, Arch. Int. Med., 1939, 64, 217). 
distribution of the current, provided this is the same for both determinations. The proportion of the total current passing through the nerve will be constant, if both the position of the electrodes and the impedance of the tissues remain unchanged between the determination of the rheobase and the threshold to an exponentially increasing current. The first of these conditions is obtained by making the two determinations in close succession. The second condition is largely satisfied by designing the stimulator so that the value of the current passed into the tissue is independent of the tissue and electrode resistances and their variations. Such a stimulator, used in the present study, has been developed and tested at the Johnson Foundation.

\section{METHOD}

One small electrode (cathode) was placed on the skin over the ulnar nerve at the elbow, and the other larger electrode made contact with the forearm. The least visible twitch of the first dorsal interosseus muscle or the least visible flexion of the distal phalanx of the thumb was used as an index of threshold stimulation of the nerve. By careful adjustment of the small electrode, one of these movements could be obtained as the only response to nerve stimulation. In any one patient, the same movement was used as the index of response throughout the experiments. The end-point was found by decreasing

TABLE I

The values of the rheobase (I0) and the threshold current

(I) for slowly rising currents of different time constants of current increase ( $\alpha$ ) were obtained by stimulation of the ulnar nerve of a normal subject

Two successive trials were made for each value of $\alpha$, using the least visible flexion of the distal phalanx of the thumb as the criterion for a threshold response. The values of the ratios $\left(R=I / I_{0}\right)$ at each value of $\alpha$ are plotted to form the accomodation curve in Figure 1.

\begin{tabular}{|c|c|c|c|}
\hline$\alpha$ & $\begin{array}{c}\mathbf{I}_{\mathbf{b}} \\
\text { (Rheobase) }\end{array}$ & I & Ratio \\
\hline milliseconds & milliamperes & milliamperes & \\
\hline 10 & $\begin{array}{l}1.45 \\
1.42\end{array}$ & $\begin{array}{l}1.95 \\
1.75\end{array}$ & $\begin{array}{l}1.34 \\
1.23\end{array}$ \\
\hline 34 & $\begin{array}{l}1.45 \\
1.40\end{array}$ & $\begin{array}{l}2.50 \\
2.20\end{array}$ & $\begin{array}{l}1.72 \\
1.57\end{array}$ \\
\hline 57 & $\begin{array}{l}1.42 \\
1.40\end{array}$ & $\begin{array}{l}2.80 \\
2.80\end{array}$ & $\begin{array}{l}1.97 \\
2.00\end{array}$ \\
\hline 78 & $\begin{array}{l}1.40 \\
1.38\end{array}$ & $\begin{array}{l}3.05 \\
3.15\end{array}$ & $\begin{array}{l}2.18 \\
2.28\end{array}$ \\
\hline 99 & $\begin{array}{l}1.38 \\
1.40\end{array}$ & $\begin{array}{l}3.30 \\
3.50\end{array}$ & $\begin{array}{l}2.40 \\
2.50\end{array}$ \\
\hline 118 & $\begin{array}{l}1.40 \\
1.40\end{array}$ & $\begin{array}{l}3.80 \\
3.80\end{array}$ & $\begin{array}{l}2.72 \\
2.72\end{array}$ \\
\hline
\end{tabular}

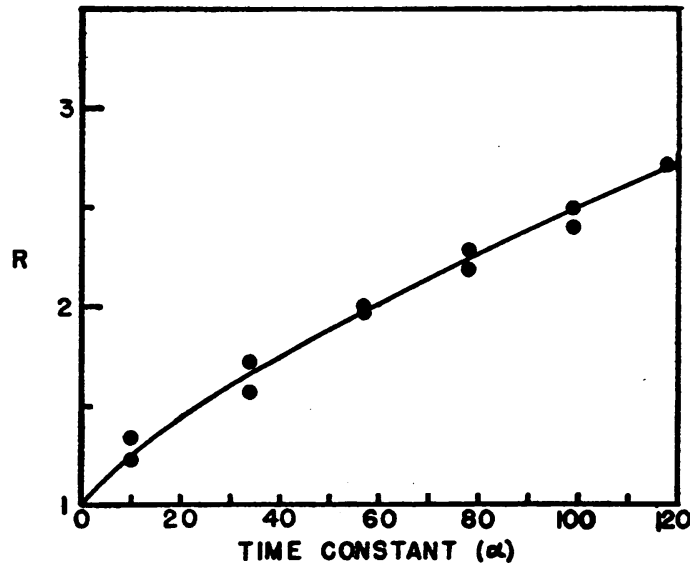

Fig. 1. A Typical Accommodation Curve for the Ulnar Nerve of a Normal Healthy Man

$R$ is the value of $I / I_{0}$ obtained from Table $I ; \alpha$ is the time constant in milliseconds for the exponentially increasing currents employed for stimulation. The contimuous line, represents the values of $R$ calculated from equation 1 using a value of $\lambda$ equal to 120 milliseconds.

a suprathreshold stimulus until the least value of current which would produce a contraction was found. Slightly higher readings were obtained when the threshold was determined by gradually increasing a. subthreshold current.

The threshold to an exponentially rising current was first obtained. Immediately thereafter, the rheobase was measured. The ratio $(R)$ of these two values of current is a measure, expressed as a multiple of the rheobase, of the proportionate elevation of the threshold (degree of accomodation) to this particular slowly rising current. Currents of various rates of rise were used. In each experiment, therefore, the threshold to each of several slowly rising currents was measured and the degree of accommodation of the nerve was expressed in each case as a multiple of the rheobase. These data can be conveniently represented by plotting the values of $R$ as a function of the time constant of the current rise $(\alpha)$. This representation is called the accommodation curve.

\section{OBSERVATIONS}

Normal subjects. Measurements typical for the normal subject are given in Table $I$ and are represented graphically in Figure 1 . The percentage difference between the pairs of ratios $(R)$ at each value of $\alpha$ illustrates the reproducibility of successive readings on the same subject. Repeated measurements on the same individual show little variation from day to day. Slight variations are occasionally found during a given day and may 


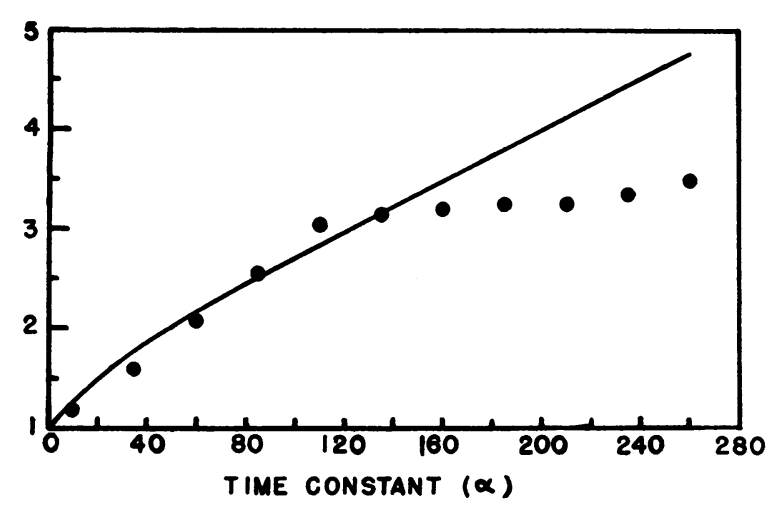

Fig. 2. The Rise in Threshold to Slowly Rising Currents (Accommodation) as Measured by the -Ratio $R$ Does not Continue to Increase for Values OF $\alpha$ GREATER than ABOUT 140 MILLISECONDS

The points represent the average of 3 separate determinations of $R$ at each value of $\alpha$. The continuous line represents the calculated accomodation curve, using a value of $\lambda$ equal to 95 milliseconds.

be related to fluctuations in the acid-base balance. ${ }^{3}$ The results obtained from a series of 15 normal individuals are similar. In no determination did the ratio $(R)$ at a value of $\alpha$ equal to 118 milliseconds exceed 3.5 or fall below 2.7 .

The value of each ratio (Figure 1) depends upon the rate of rise of the stimulating current, as determined by $\alpha$, and upon the rate of rise of the threshold of the nerve. The latter is regulated by a time constant $\lambda$ which is characteristic of the nerve. The relation between $R, \alpha$, and $\lambda$ has been derived by Hill (2).

$$
R=\frac{I}{I_{0}}=\left(\frac{\alpha}{\lambda}\right)^{\alpha /(\alpha-\lambda)}
$$

The continuous line (Figure 1) represents the curve obtained from this equation using a value of $\lambda$ equal to 120 milliseconds. The agreement between theory and experiment is good up to a value of $\alpha$ equal to 118 milliseconds.

When currents with slower rates of rise were used, no considerable further increase of the threshold was noted. The points in Figure 2 are the average of 3 measurements of $R$ at each of the values of $\alpha$ indicated on the abscissae. The line represents the calculated values for a $\lambda$ value equal to 95 milliseconds. There is considerable

\footnotetext{
3 In latent tetany, these variations become of enough significance to be misleading unless readings are taken at the same time of the day, or several times during the same day.
}

variation of the experimental values from the theoretical above values of $\alpha$ equal to 140 milliseconds. The "coefficient of accommodation" $(\lambda)$ does not represent the experimental results except within a restricted range of values for $\alpha$. Therefore, in the subsequent figures, either the complete experimental curve or the ratio $(R)$ at 118 milliseconds is employed to show the changes in accommodation.

Subjects with parathyroid deficiency. The measurements of accommodation on these subjects during the control period were within the normal range. After removal of the calcium and viosterol therapy, the ratios $(R)$ at each time constant $(\alpha)$ became smaller than normal on the first or second day, fluctuated at markedly subnormal values, and then rose rapidly after the administration of A T 10 to values above those observed during the "normal" control period.

Case 1. L. H., aged 42, thyroidectomy in 1923; symptoms of parathyroid deficiency for 15 years, relieved irregularly by parathormone, and calcium and viosterol therapy.

In Figure 3 is represented the response in this patient to withdrawal of calcium. This result is typical of all the patients with hypoparathyroidism who were tested.

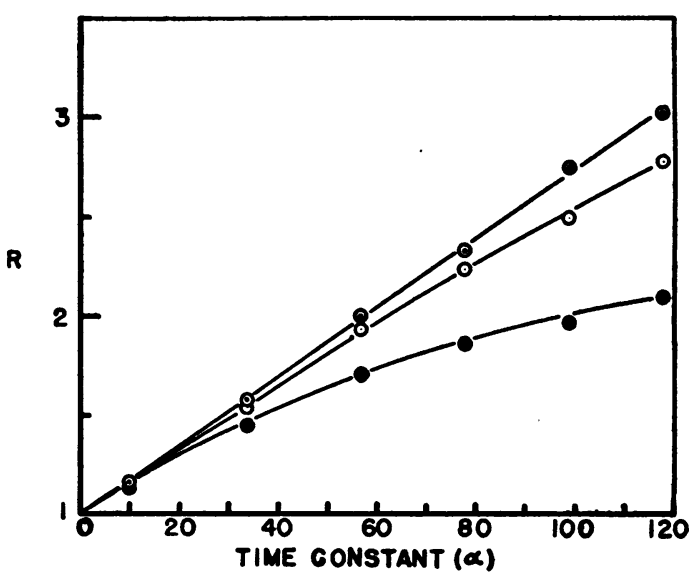

Fig. 3. Case 1. The Change in the accommodation Curve Observed When the Level of the Blood Serum Calcium is Falling After Discontinuing Calcium Therapy in a Patient with Parathyoid DEFICIENCY

Values of $\alpha$ are in milliseconds.

$\odot=$ ratios at the 6 values of $\alpha$ before therapy was discontinued (Nov. 14).

$\mathrm{O}=$ ratios observed on the first day without calcium therapy (Nov. 15).

- = ratios observed on the fourth day without calcium therapy (Nov. 18). 
Calcium lactate and viosterol had been given up to and including November 14th. On November 15th, no calcium was given, and the values of $R$ for each of the slowly rising currents were found to be less than the control values. At this time, there were no clinical symptoms or signs of increase in nerve irritability. The measurements on November 18th, the fourth day without therapy, revealed a marked decrease in the rate of accomodation as indicated by values of $R$ much lower than those obtained during the control period. By this time, clinical examination revealed early signs of increased nerve irritability.

In Figure 4, the response to administration of A T 10 is charted. No therapy was given from November 15 th to 26 th, inclusive. The values of the ratios measured on November 26th remained markedly reduced. On the morning of November 27th, A T 10 was administered, and $2 \frac{1}{2}$ hours later, there was an increase in each of the values of $R$, indicating an increased ability of the nerve to accommodate. At this time, there was no clinical evidence of improvement. Further records taken on the third and sixth days of A T 10 administration are shown, each illustrating additional improvement in the physiological state of the nerve as determined by this method. The record of December 1st indicates a degree of accommodation even greater than that in the normal

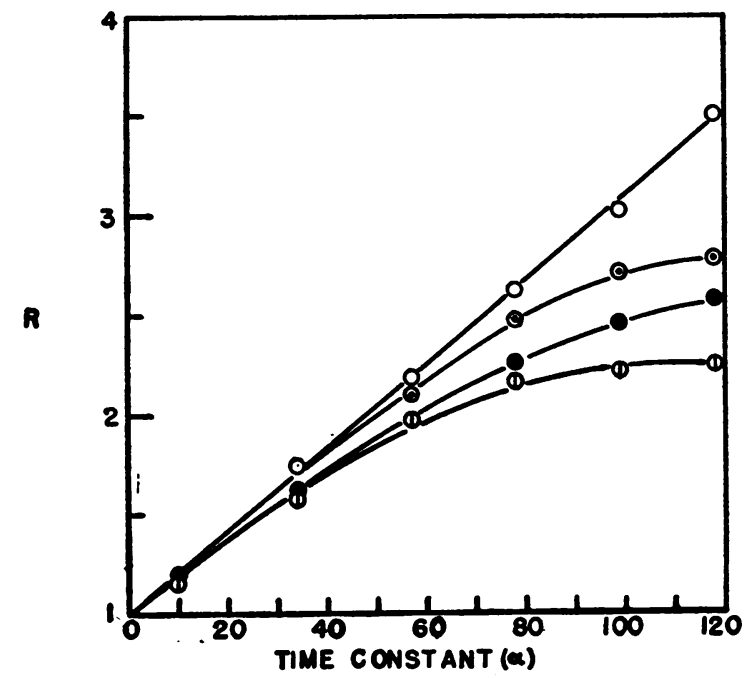

Fig. 4. Case 1. The Change in the accommodation Curve Observed During the Rise in the Levei of Serum Calcium After Administration of DihydroTACHYSTEROL (A T 10)

Values of $\alpha$ in milliseconds.

$\Phi=$ Values of $R$ on Nov. 26th-twelfth day without therapy.

$=$ Values of $R$ on Nov. 27th-21/2 hours after administration of A T 10.

$\odot=$ Values of $R$ on Nov. 29th-the third day of A T 10 administration.

$\mathrm{O}=$ Values of $R$ on Dec. 1st-the fifth day of A T 10 administration.

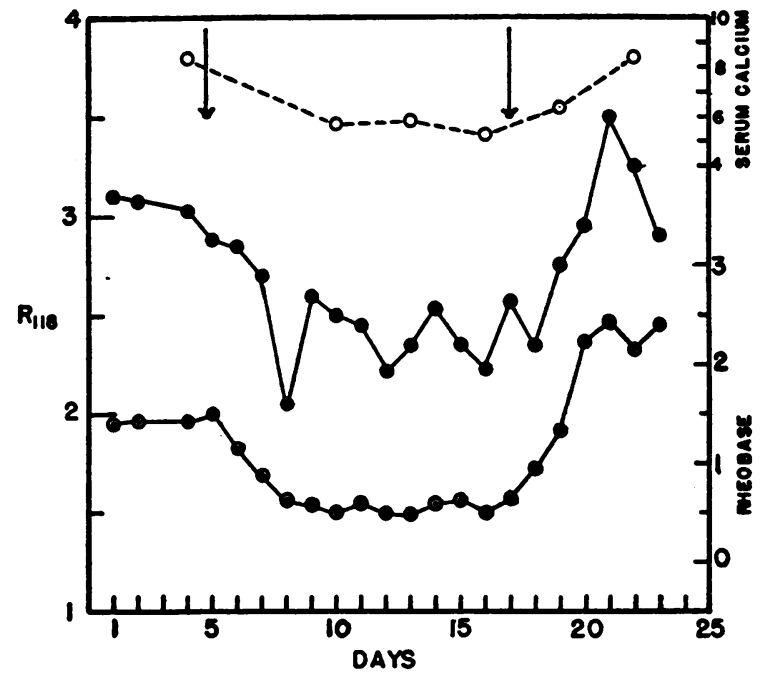

Fig. 5. Case 1. Chart Showing Relationship of Serum Calctum Values, Rheobase Values, and the Values of the Rate of Accomodation (as Represented by the Value of the Ratio When $\alpha$ is Equal to 118 MILLISECONDS)

The first arrow indicates the time of withdrawal of maintenance dosage of calcium and viosterol. The second arrow indicates the beginning of administration of A T 10 .

subjects. Smaller doses of A T 10 were given thereafter, and subsequent observations showed a return of the nerve processes to the normal state.

The relation of the ratios to the level of the blood serum calcium is shown in Figure 5 . The degree of accomodation is represented by the value of the ratio when $\alpha$ is equal to 118 milliseconds. The total serum calcium is measured in milligrams per cent.4 The changes in the degree of accommodation upon withdrawal of calcium and upon administration of A T 10 parallel the changes in the blood calcium. Included in Figure 5 are the average values of the rheobase measured during these experiments. The change in the stability of the nerves, indicated by these threshold values of direct current, also parallels the change in blood calcium.

Case 2. M. H., aged 30 years; thyroidectomy July 1935; hypoparathyroid tetany appeared on the following day and was subsequently forestalled with moderate success for $2 \frac{1}{2}$ years by the use of calcium, viosterol, and parathyroid hormone. Studies were started February 10, 1938; therapy discontinued after February 13th; A T 10 given, starting February 20th.

The withdrawal of calcium and viosterol was followed by a marked decrease in the rate of accommodation. After administration of A T 10 , restoration of $R$ to values above the normal was observed. The changes in

4 The ratio of diffusible calcium to total calcium was approximately constant for each subject. The diffusable calcium was about equal to the calculated calcium ion concentration. (See paper by E. Rose and F. W. Sunderman for details.) 


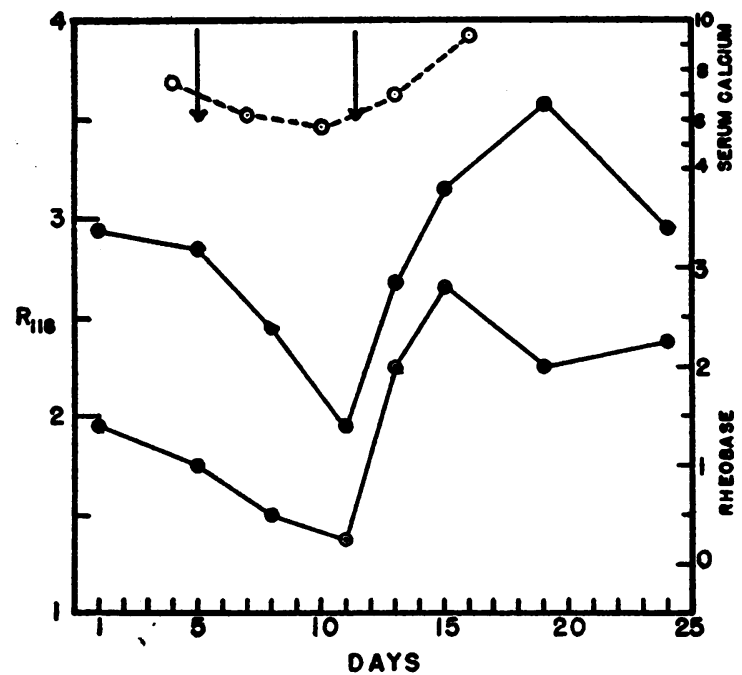

Fig. 6. Case 2. Illustrating the Relationship of Serum Calctum, Rheobase, and Ratios at 118 milliseconds, in Another Case of Parathyroid Deficiency

the blood calcium level were similar to those in the first case. These changes are illustrated in Figure 6.

Case 3. J. J., aged 38 years; hypoparathyroid symptoms for 10 years following thyroidectomy, partially relieved by calcium and viosterol. Studied in September, 1937; calcium withdrawn September 14th; A T 10 given September 21st.

When the calcium was discontinued, there was a very marked decrease in the rate of accomodation. After administration of A T 10, the rate of accomodation increased as usual from an abnormally low figure to a value at the lower limits of the normal range. The changes in the level of blood calcium during this period were similar to those previously noted.

Case 4. H. E., aged 39 years; post-thyroidectomy hypoparathyroidism had been present for 20 years in this patient. Symptoms varied but were usually relieved by the administration of calcium, although the blood calcium remained persistently low (6 to $8 \mathrm{mgm}$. per cent). Studied December 1937; calcium withdrawn December 13th; A T 10 given December 20th.

At the end of the control period, the values of the ratio at each time constant were normal, yet at this time the blood serum calcium value was quite low (7.9 mgm. per cent). After withdrawal of therapy, the decrease in the rate of accomodation was moderate and not as abrupt as in the other cases. The blood calcium level also fell but moderately (to $6.8 \mathrm{mgm}$. per cent on the sixth day.) After administration of A T 10, there was a marked rise in the values for the rate of accommodation, and the blood calcium.

Case 5. M. R., aged 29; subtotal thyroidectomy, September 1937; subacute symptoms of hypothyroidism and hypoparathyroidism after operation. Admitted to the hospital in January 1938 for study. This patient had not been receiving substitution therapy. Therefore, the initial observations were made at a time when the blood calcium was abnormally low.

During this period without therapy, the rate of accomodation was also found to be abnormally low. After administration of A T 10, the rate of accommodation and the level of serum calcium increased in parallel fashion. The rheobase also rose markedly.

Several evidences of functional changes in the central nervous system are worthy of mention. In all cases, the tendon reflexes were hypoactive. Three cases complained of being unduly apprehensive and fearful, particularly during the periods when the blood calcium was low. One noted impairment of recent memory during the hypocalcemic periods.

\section{DISCUSSION}

The studies of subjects with parathyroid deficiency indicate that characteristic alterations of nerve function accompany changes in the blood calcium level and that these can be expressed quantitatively by measurement of the degree of accommodation of the nerve.

It is to be noted, however, that a given accommodation curve cannot be considered as characteristic of a given value of the serum calcium. A normal accomodation curve may be obtained when the blood calcium is relatively low, if this low value has been present for a considerable time. Presumably, a gradual physiological adjustment to this low level may take place. In contrast to such long-time adjustments, every rise and fall of the level of the blood calcium taking place within a period of several days is accompanied by corresponding changes in the rate of accomodation. These changes are measurable before clinical signs and symptoms of the altered blood chemistry are apparent.

The critical value of the threshold stimulus is clearly defined. It was noted that, as successively more slowly rising currents were used, the muscular response became progressively slower in onset and continued in partial tetanus during the flow of the current. A slow contraction rather than a clear-cut twitch is then observed. In the subjects with low blood calcium and slower accommodation, the slow onset and tetanic nature of the contraction occurred with definitely lower values of current strength and with more rapidly rising currents. This is another indication of the reduced ability of the nerves in such subjects to accommodate to the electrical stimuli. 
One theory (2) predicts an accommodation curve which gradually approaches a limiting straight line of finite slope. The observed curves follow, in general, the predicted course up to values of $\alpha$ equal to 118 milliseconds. Beyond this point, the curve becomes nearly horizontal (Figure 2). This form of curve has also been found in an earlier investigation (3). It would seem that not only does accommodation proceed at a certain rate, but also that the threshold reaches a certain value beyond which it rises but slowly or not at all. For these reasons, as was previously noted, the index of the rate of accomodation used was the graphic representation of the entire set of ratios. By the comparison of a series of determinations, it was found that the value of the ratio obtained when $\alpha$ equaled 118 milliseconds represented faithfully the variations shown by the total curve (Figures 5 and 6). Using either of these indices, it has been found that normal values fall within a limited range and that the amount of variation in repeated determinations is about \pm 5 per cent of the average value.

The well-known alterations in the threshold of the nerve to abruptly rising currents (rheobase) associated with a falling or rising blood calcium level were observed in these studies. This change is an additional indication of the altered physiological state of the nerve. The values of the observed rheobase, however, were found to be subject to considerable variation, depending upon the position of the stimulating electrode and probably the thickness of the skin. In contrast, deliberate or accidental changes in the apparent rheobase, within certain limits, failed to alter the observed values of $R$ provided the two determinations of threshold used to calculate $R$ were made under comparable conditions.

It is to be noted that the change in the rheobase due to alteration in the blood calcium does not account for the changes in accommodation. A decrease in the rheobase, when the blood calcium decreases, would tend to increase the value of $R$, whereas, it is observed that $R$ also decreases under such conditions.

These observations show that a quantitative objective measure of a physiological change in the nerve tissue of patients with hypoparathyroid disease can be obtained. It is thus possible accurately to compare the changes in blood cal- cium with the altered functional condition of the nerve tissue. This measure of the condition of the nerves was just as adequate as an index of the response to A T 10 of patients with parathyroid deficiency as was the measure of the concentration of blood calcium. The procedure could be employed to determine the amount of A T 10 (or other medication) required to maintain normal the properties of nerve tissue.

\section{SUMMARY}

1. A measure of the property of accommodation in human peripheral nerve can be readily obtained with consistent results.

2. The method used has the advantage of being relatively independent of certain variable factors which may impair the accuracy of quantitative measurements of peripheral nerve irritability made through the intact skin.

3. In agreement with previous observations (3), the accommodation curve for human nerve is found to be terminated by a horizontal portion which is probably associated with repetitive firing in the nerve fibers.

4. In 5 cases of hypoparathyroidism, the functional state of the peripheral nerve as indicated by measures of the property of accommodation was found to vary characteristically with changes in the calcium ion concentration in the blood.

5. This method of studying this disease is useful whenever a detailed quantitative measure of the changes in the state of the nerve tissue is desired. The quantitative measure of the functional change makes possible a relatively accurate comparison of the degree of change in the nerve tissue with the degree of chemical change in the blood and tissue fluids.

The authors wish to acknowledge their indebtedness to Professor D. W. Bronk for suggesting their collaboration in this research.

\section{BIBLIOGRAPHY}

1. Solandt, D. Y., The measurement of "accommodation" in nerve. Proc. Roy. Soc., London, s.B., 1936, 119, 355.

2. Hill, A. V., Excitation and accommodation in nerve. Proc. Roy. Soc., London, s.B., 1936, 119, 305.

3. Liberson, W., Quelques observations sur l'excitation des nerfs et des muscles de l'homme par les courants lentement croissants. Compt. rend. Soc. de biol., 1934, 116, 1319. 